\title{
Unusual Adverse Event of Tetanus: Rectus Sheath Hematoma
}

\author{
Fumiya Inoue, Toshihisa Ichiba and Hiroshi Naitou
}

\begin{abstract}
:
Tetanus is a nervous system disorder characterized by muscular spasms and autonomic hyperactivity, such as unstable blood pressure. We herein report a case of tetanus in a patient in shock complicated with a rectus sheath hematoma caused by rupture of a pseudo-aneurysm of the inferior epigastric artery. A rectus sheath hematoma might be misdiagnosed as unstable blood pressure associated with autonomic hyperactivity, which is usually observed in patients with tetanus. The possibility of the occurrence of bleeding complications should be considered if a patient with tetanus has severe and persistent blood pressure reduction.
\end{abstract}

Key words: autonomic hyperactivity, muscular spasm, rectus sheath hematoma, tetanus

(Intern Med 60: 151-153, 2021)

(DOI: 10.2169/internalmedicine.4800-20)

\section{Introduction}

Tetanus is a nervous system disorder characterized by muscular spasms and autonomic hyperactivity that is caused by the toxin produced by Clostridium tetani $(1,2)$. A diagnosis of tetanus is often based on typical clinical findings. However, tetanus in the presence of protective concentrations of antibody is rare, so serum antibody titers are useful for ruling out a diagnosis of tetanus (3).

Autonomic hyperactivity such as unstable blood pressure is a well-known complication. A reduction in blood pressure due to bleeding complications may therefore be considered part of the natural course of autonomic hyperactivity, resulting in a delayed diagnosis of critical bleeding events in patients with tetanus.

We herein report a case of tetanus in a patient in shock complicated with a rectus sheath hematoma caused by rupture of a pseudo-aneurysm of the inferior epigastric artery.

\section{Case Report}

An 82-year-old woman with a history of hypothyroidism complained of an articulatory disorder. Ten days before admission, she had injured her bilateral first toes. On day 4, trismus, risus sardonicus, dysphagia and opisthotonus ap- peared. We therefore made a diagnosis of tetanus based on these typical clinical findings.

Treatment with tetanus toxoid, tetanus immunoglobulin and metronidazole was started in the intensive-care unit. She was treated with intravenous diazepam and magnesium sulfate $\left(\mathrm{MgSO}_{4}\right)$ for muscle spasms and autonomic hyperactivity, and 10,000 units of prophylactic heparin was administered intravenously, maintaining an activated partial thromboplastin time (aPTT) around 45.0 seconds to prevent venous thromboembolism.

Although muscle spasms and autonomic hyperactivity were controlled well, she suddenly became hemodynamically unstable on day 19 . We initially considered the instability in blood pressure to be a result of tetanus-associated autonomic hyperactivity, but we found that the hemoglobin level had decreased from $8.9 \mathrm{~g} / \mathrm{dL}$ initially to $5.8 \mathrm{~g} / \mathrm{dL}$ at 10 hours after this event. We therefore performed abdominal contrast-enhanced computed tomography (CT), which revealed a left-sided rectus sheath hematoma with extravasation of contrast media (Figure A). Despite fluid resuscitation and transfusion, she was still hemodynamically unstable, and we decided to perform percutaneous arterial embolization toward a ruptured inferior epigastric arterial pseudoaneurysm (Figure B). After successful embolization, she became hemodynamically stable and was discharged on day 97. 


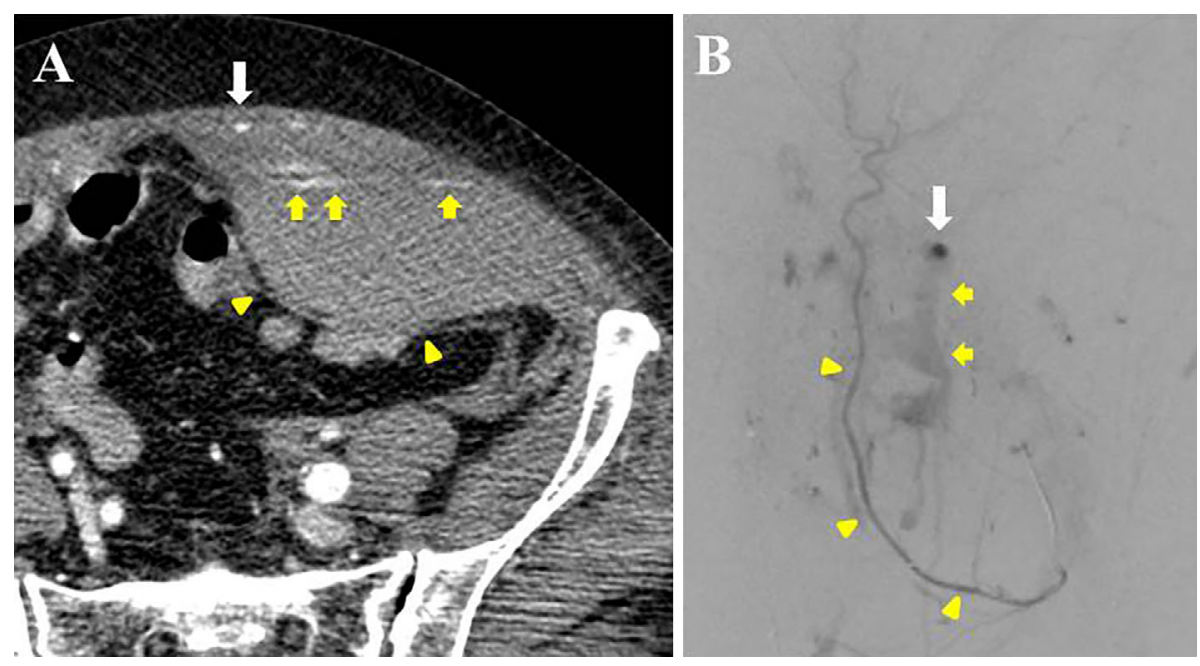

Figure. (A) CT revealed rectus sheath hematoma (arrowheads), dilated inferior epigastric artery (white arrow) and pooling of contrast media in the rectus sheath (yellow arrows). (B) Angiography revealed an inferior epigastric artery (arrowheads) pseudo-aneurysm (white arrow) and extravasation of contrast media from the ruptured pseudo-aneurysm (yellow arrows).

\section{Discussion}

The course of the patient indicated two important clinical issues: 1) tetanus is occasionally complicated with a rectus sheath hematoma caused by muscular spasms, and 2) a rectus sheath hematoma might be diagnosed as unstable blood pressure associated with autonomic dysfunction, which is usually observed in patients with tetanus.

Rectus sheath hematoma is an uncommon clinical condition caused by bleeding from epigastric arteries into the rectus sheath and is usually associated with abdominal trauma and anticoagulation (4). Most patients complain of abdominal pain and tenderness and are treated conservatively. There has been only one case report of tetanus with a rectus sheath hematoma that was treated conservatively (5). Other sites, such as iliopsoas hematoma and oral hematoma, are rarely reported $(6,7)$. Our case is the first reported case of tetanus-associated rectus sheath hematoma with a hemodynamically unstable condition that was treated by percutaneous arterial embolization.

Muscle spasm is the main characteristic of tetanus and is life-threatening. Sedative and neuromuscular blocking agents are therefore often used to control muscular spasms. According to these treatments, about half of the patients require mechanical ventilation, and we need to use prophylactic anticoagulation for venous thromboembolism (2). We speculated that direct damage to small vessels caused by muscular spasms and anticoagulation might be the underlying mechanisms of pseudo-aneurysm formation in the inferior epigastric artery, leading to rupture into the rectus sheath and shock in the present patient (5). Our patient was hemodynamically unstable, and extravasation of the inferior epigastric artery was revealed by CT. We therefore decided to perform percutaneous arterial embolization. According to a lit- erature review, hemorrhaging ceased in all patients, and 75\% of the patients had a favorable course of hospital discharge following treatment with percutaneous arterial embolization (8). Surgical ligation is limited because it is difficult to identify the bleeding site, so percutaneous arterial embolization should be used to treat rectus a sheath hematoma in patients who remain hemodynamically unstable despite conservative management. The baseline aPTT was controlled adequately around 40.0 seconds, but the aPTT after the bleeding event was prolonged to 70.0 seconds. Patents with tetanus may be complicated with rare but serious bleeding complications, so the use of prophylactic heparin should be considered carefully.

Unstable blood pressure is the main feature of autonomic hyperactivity in patients with tetanus and ranges from a minimum systolic blood pressure of $60 \mathrm{mmHg}$ to a maximum of $290 \mathrm{mmHg}$ (9). However, most patients are sedated to control muscular spasms and thus do not complain of abdominal pain. As a result, we tend to consider a blood pressure reduction caused by bleeding complications, such as a rectus sheath hematoma, as part of the natural course of autonomic hyperactivity, resulting in a delayed diagnosis of critical bleeding events in patients with tetanus. Indeed, we initially assumed that the blood pressure reduction in our patient had been caused by autonomic hyperactivity and administered diazepam and $\mathrm{MgSO}_{4}$.

Muscular spasms and autonomic hyperactivity are wellknown symptoms of tetanus and tend to receive focus. However, patients with tetanus usually need treatment in an intensive-care unit for about one month, and various direct or indirect tetanus-associated complications can occur during the treatment period. The possibility of bleeding complications, such as a rectus sheath hematoma caused by muscular spasms, should be considered if a patient with tetanus has severe and persistent blood pressure reduction in order to 
avoid a misdiagnosis of autonomic hyperactivity.

The authors state that they have no Conflict of Interest (COI).

\section{References}

1. Afshar M, Raju M, Ansell D, Bleck TP. Narrative review: tetanusa health threat after natural disasters in developing countries. Ann Intern Med 154: 329-335, 2011.

2. Nakajima M, Aso S, Matsui H, Fushimi K, Yasunaga H. Clinical features and outcomes of tetanus: analysis using a National Inpatient Database in Japan. J Crit Care 44: 388-391, 2018.

3. Yen LM, Thwaites CL. Tetanus. Lancet 393: 1657-1668, 2019.

4. Hatjipetrou A, Anyfantakis D, Kastanakis M. Rectus sheath hematoma: a review of the literature. Int J Surg 13: 267-271, 2015.

5. Suhr GM, Green AE Jr. Rectus abdominis sheath hematoma as a complication of tetanus. Diagnosis by computed tomography scan- ning. Clin Imaging 13: 82-86, 1989.

6. Ishii N, Mochizuki H, Shiomi K, Nakazato M. Iliopsoas hematoma as a complication of tetanus. Neurol Clin Neurosci 7: 26-30, 2019.

7. Mayo-Yáñez M, Costa LI. Trismus and oral haematoma, a rare presentation of tetanus. Acta Otorrinolaringol Esp 71: 129-130, 2020.

8. Rimola J, Perendreu J, Falco J, Fortuno JR, Massuet A, Branera J. Percutaneous arterial embolization in the management of rectus sheath hematoma. AJR Am J Roentgenol 188: 497-502, 2007.

9. Gregorakos L, Kerezoudi E, Dimopoulos G, Thomaides T. Management of blood pressure instability in severe tetanus: the use of clonidine. Intensive Care Med 23: 893-895, 1997.

The Internal Medicine is an Open Access journal distributed under the Creative Commons Attribution-NonCommercial-NoDerivatives 4.0 International License. To view the details of this license, please visit (https://creativecommons.org/licenses/ by-nc-nd/4.0/).

(C) 2021 The Japanese Society of Internal Medicine Intern Med 60: 151-153, 2021 